\title{
Is fetal nuchal cord associated with autism spectrum disorder? A meta-analysis
}

Ensiyeh Jenabi, $\mathrm{PhD}^{1}$, Maryam Ahmadi, $\mathrm{MD}^{2}$, Azam Maleki, $\mathrm{PhD}^{3}$

${ }^{1}$ Autism Spectrum Disorders Research Center, Hamadan University of Medical Sciences, Hamadan, Iran; ${ }^{2}$ Clinical Research Development Unit of Fatemieh Hospital, Department of Gynecology, School of Medicine, Hamadan University of Medical Sciences, Hamadan, Iran; ${ }^{3}$ Social Determinants of Health Research Center, Zanjan University of Medical Sciences, Zanjan, Iran

Purpose: There is inconsistent evidence about the association between fetal nuchal cord and the risk of autism spectrum disorder (ASD). We performed a meta-analysis to investigate whether fetal nuchal cord is associated with an increased risk of ASD in offspring.

Methods: Three main English databases were searched until January 2021. The Newcastle-Ottawa Scale was used to assess study quality. Study heterogeneity was determined using the $I^{2}$ statistic, while publication bias was assessed using Begg and Egger tests. Results are presented as odds ratios (ORs) and relative ratios with 95\% confidence intervals (CI) and were determined by a random-effects model.

Results: Five articles (1 cohort, 4 case-control; 3,088 total children) were included in the present meta-analysis. Fetal nuchal cord was not a risk factor for ASD (OR, 1.11; 95\% CI, 0.66-1.57). There was homogeneity among studies that reported a risk of ASD $\left(I^{2}=0.0 \%\right)$.

Conclusion: Our results showed that fetal nuchal cord is not a risk factor for ASD. Future large cohort studies should confirm this finding.

Key words: Autism spectrum disorder, Fetal nuchal cord, Meta-analysis

\section{Key message}

Question: Is fetal nuchal cord a risk factor for autism spectrum disorder (ASD)?

Finding: Five articles (1 cohort, 4 case-control; total 3,088 children) were included in the present meta-analysis. Fetal nuchal cord was not a risk factor for ASD (odds ratio, 1.11; 95\% confidence interval, 0.66-1.57). There was homogeneity among studies that reported a risk of ASD $\left(I^{2}=0.0\right)$.

Meaning: Fetal nuchal cord is not a risk factor for ASD.

\section{Introduction}

Autism spectrum disorder (ASD) is a developmental disorder that features severe impairments with social interaction and communication as well as stereotypic behavior. Its prevalence is 1-2 in per 100 individuals. ${ }^{1)}$ ASD often develops in the second year of life, and its symptoms usually appear around 3 years of age..$^{2)}$ Although etiology of ASD remains unclear, both genetic and environmental factors are presumed involved in its disease development. ${ }^{3)}$

Researchers have reported that prenatal and perinatal risk factors, including preeclampsia, preterm labor, assisted reproductive technology, small for gestational age, neonatal jaundice, and low birth weight (LBW), contribute to the development of ASD. ${ }^{4-6)}$

Nuchal cord is defined as the umbilical cord being wound around the fetal neck. ${ }^{7}$ This phenomenon increases the risk of fetal distress and is usually associated with a long umbilical cord. ${ }^{8)}$ The prevalence of nuchal cord is about 10\%-29\% of fetuses and increases with increasing gestational age. ${ }^{9}$ Nuchal cord can lead to acidosis, an increased need for cesarean section, a low Apgar score, growth restriction, and severe spastic cerebral palsy. ${ }^{9)}$

Studies have investigated the association between fetal nuchal cord and ASD in children. ${ }^{3,10,11)}$ On reported that fetal nuchal cord was associated with ASD, ${ }^{3)}$ while others did not. ${ }^{10,11)}$ Moreover, there are limited reviews on this topic. ${ }^{12-14)}$ Wang et al. ${ }^{13)}$ reported that the results of 2 studies with high heterogeneity used for determining an increased risk of ASD with fetal nuchal cord or other umbilical cord events were analyzed as a subgroup. Accordingly, here we performed a meta-analysis to further investigate the association between fetal nuchal cord and ASD among children and conducted a systematic review of observational studies to assess this association.

\footnotetext{
Corresponding author: Azam Maleki, PhD. Second Building of Vice Chancellor for Research and Technology, Social Determinants of Health Research Center, Zanjan University of Medical Sciences, Zanjan, Iran

凶Email: malekia@zums.ac.ir, malekia41@yahoo.com, https://orcid.org/0000-0001-7888-1985

Received: 21 April, 2021, Revised: 26 August, 2021, Accepted: 13 September, 2021

This is an open-access article distributed under the terms of the Creative Commons Attribution Non-Commercial License (http://creativecommons.org/licenses/by$\mathrm{nc} / 4.0 /$ ) which permits unrestricted non-commercial use, distribution, and reproduction in any medium, provided the original work is properly cited.

Copyright (c) 2022 by The Korean Pediatric Society
} 


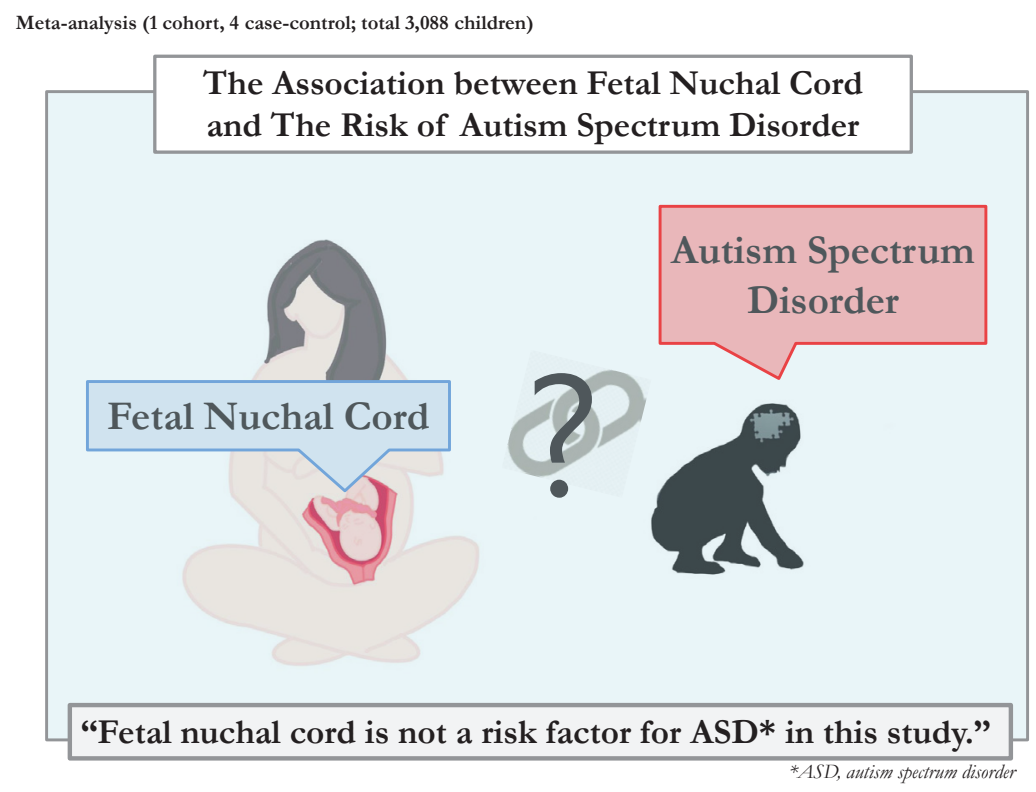

\section{Graphical abstract}

\section{Methods}

\section{Data sources}

This meta-analysis was performed and reported in adherence with the PRISMA (Preferred Reporting Items for Systematic Reviews and Meta-analysis) 2020 checklist. ${ }^{15)}$

\section{Search strategy}

We systematically searched PubMed, Scopus, and the Web of Science for studies published through January 2021. We did not restrict publication language or date. The search algorithm was performed as follows: (fetal nuchal cord OR nuchal cord OR umbilical cord around neck) AND (autism spectrum disorder OR autism spectrum disorders OR ASD).

\section{Inclusion and exclusion criteria}

Inclusion criteria for the systematic review study were: case-control or cohort study; diagnosis of ASD based on the Diagnostic Statistical Manual of Mental Disorders, fourth or fifth revision, International Classification of Diseases, eighth, ninth, or tenth revision, or Child Autistic Rating Scale; and metaanalysis exposure of fetal nuchal cord and output of risk of ASD. The exclusion criterion was study design of case report, case series, or review or article type of editorial or letter to the editor.

\section{Data extraction}

Two investigators independently reviewed the article titles and abstracts and performed the full-text reviews. The reference lists of the included studies were manually assessed to identify potential studies that may not have been retrieved from the databases.

The data were extracted independently by 2 investigators (EJ and $(\mathrm{AM})$ and included the following: first author, publication year, study design, number of participants, association between fetal nuchal cord and ASD, odds ratio (OR), hazard ratio (HR), associated 95\% confidence interval (CI), adjustment, child age (range/mean), and study quality.

\section{Quality assessment}

The modified Newcastle-Ottawa Scale was used to assess observational study quality. ${ }^{16)}$ This scale includes participant selection, comparability of ASD and non-ASD children, and outcome assessment. There were 9 total points on the quality scale. Studies with $\geq 7$ points were considered of high quality. Two investigators (EJ and AM) independently performed the quality assessment.

\section{Statistical analysis}

Data are expressed as OR or HR with 95\% CI to assess the association between fetal nuchal cord and the risk of ASD. An $I^{2}>75 \%$ based on Higgins et al. $(2003)^{17)}$ was considered to indicate high heterogeneity. Publication bias was assessed using Begg and Egger regression tests. ${ }^{18)}$ Significance was set at $P<0.05$. Analyses were performed using Stata 16 (StataCorp, College Station, TX, USA).

\section{Results}

\section{Study description}

The initial search yielded 263 studies; of them, 170 were excluded as duplicates. Next, 82 and 6 studies were excluded after the title/abstract and full-text screening, respectively. After the full-text screening, 3 studies did not meet the inclusion criteria, 2 were reviews, and 1 was a commentary.

Ultimately, 5 studies that met the inclusion criteria were included in this meta-analysis, including 1 cohort study ${ }^{11)}$ and 4 case-control studies ${ }^{3,10,19,20)}$ with a total sample size of 3,088 children. Fig. 1 presents a patient selection flow chart. 


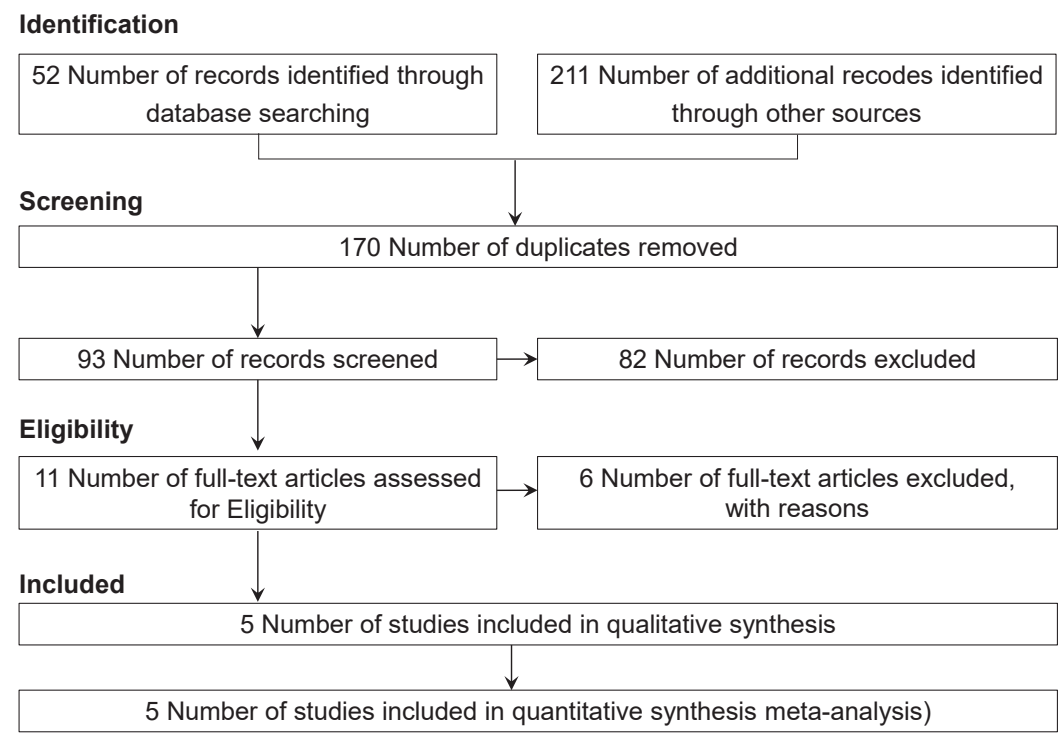

Fig. 1. The selection ow chart of studies included in the systematic review.

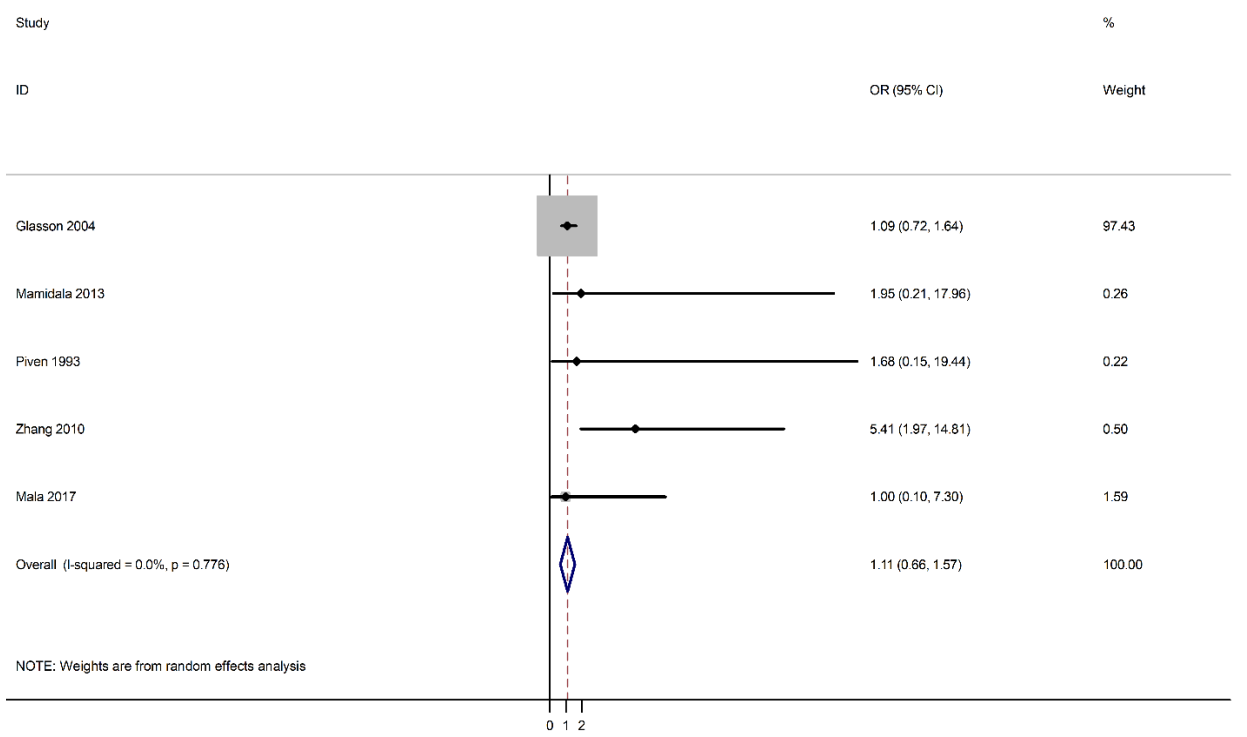

Fig. 2. Forest plot of the association between fetal nuchal cord and autism spectrum disorder. OR, odds ratio; $\mathrm{Cl}$, confidence interval.

The confounding variables for the association between fetal nuchal cord and ASD were maternal age, child's sex, race/ethnicity, education, parity, smoking, prenatal care, year of diagnosis, and psychosocial disorder during pregnancy.

\section{Main analysis}

As shown in Fig. 2, fetal nuchal cord was not a risk factor for ASD (OR, 1.11; 95\% CI. 0.66-1.57). The results of the studies were consistent and lacked heterogeneity in reporting the risk of $\operatorname{ASD}\left(I^{2}=0.0 \%\right)$.

The results reported that no significant publication bias was observed between fetal nuchal cord and ASD according to the Begg and Egger tests $(P=0.624$ and $P=0.438$, respectively).

\section{Sensitivity analysis}

We explored the impact of one low-quality study by using a sensitivity analysis and excluding that study. ${ }^{20)}$ Regardless, fetal nuchal cord was not a risk factor for ASD (OR, 1.11; 95\% CI, $0.66-1.57)$. There was homogeneity among the studies that reported a risk of ASD $\left(I^{2}=0.0\right)$.

\section{Study quality}

In the present meta-analysis, 4 studies were of high quality and one was of low quality (Table 1 ).

\section{Discussion}

To our knowledge, this is the first systematic review and 
Table 1. Results reported by the studies included in the meta-analysis

\begin{tabular}{llclcccc}
\hline Study & Design & Sample size & Estimate & Adjustment & Age range (yr) & Autism criteria & Quality \\
\hline Glasson et al., ${ }^{19)}$ 2004 & Case-control & 1,778 & Odds ratio & Crude & $<3$ & DSM & High \\
Mamidala et al.., ${ }^{11)} 2013$ & Cohort & 942 & Odds ratio & Crude & $<10$ & CARS & High \\
Piven et al., ${ }^{20)} 1993$ & Case-control & 73 & Odds ratio & Crude & $5-25$ & ICD-10 & Low \\
Zhang et al.., ${ }^{3)} 2010$ & Case-control & 190 & Odds ratio & Adjusted & $3-21$ & ICD-10, CARS & High \\
Malla et al.., ${ }^{10)}$ 2017 & Case-control & 105 & Odds ratio & Crude & $3-5$ & ICD-10, DSM-IV & High \\
\hline
\end{tabular}

DSM, Diagnostic and Statistical Manual of Mental Disorders; ICD-10, International Statistical Classification of Diseases, 10th revision; CARS, Child Autistic Rating Scale; DSM-IV, Diagnostic and Statistical Manual of Mental Disorders, 4th edition.

meta-analysis to assess the association between fetal nuchal cord and the risk of ASD. Based on the available evidence, the metaanalysis showed that fetal nuchal cord was not a risk factor for ASD. In addition, the $I^{2}$ value was $0 \%$; therefore, the results of the studies were consistent and interstudy heterogeneity may have been negligible.

According to this evidence, nuchal cord may not directly increase the risk of ASD in offspring, but its complications may have long-term effects on infant health and contribute to an increased risk of ASD. ${ }^{21)}$ Two types of nuchal cords have been identified at delivery. Type A encircling the neck in an unlocked pattern. Type B encircling the neck in a locked pattern. Symptomatic nuchal cord is classified as type $\mathrm{B}$, which is identified as being extremely tight and encircling the neck in a locked pattern. Perinatal distress, LBW, breech presentation, and cesarean section are associated with type B nuchal cord.9" Babies with nuchal cord more often require neonatal intensive care unit admission, develop fetal acidemia, and require resuscitation. ${ }^{22)}$

A small number of studies have shown that hypoxemia with or without meconium-stained amniotic fluid in conjunction with nuchal cord can lead to central nervous system damage in the newborn, who may demonstrate subclinical deficits in performance and development at 1 year of age. ${ }^{9,23)}$ The risk of ASD may increase among offspring whose mothers undergo a cesarean section, experience preterm labor, or face fetal malpresentation. ${ }^{24-26)}$

Karabeg et al. ${ }^{23)}$ showed a significant association between a tight cord around the neck and neurodevelopmental outcomes. They showed that a higher degree of severity resulted in greater neurodevelopmental deviation. However, they did not investigate the association between nuchal cord and the risk of ASD.

Our study has some limitations. First, there were a limited number of available studies on the association between fetal nuchal cord and the risk of ASD. Second, in this meta-analysis, one study was adjusted for some risk factors and 4 studies reported crude data. In most of the included studies, the followup period was very short, and the time of the event and status of asphyxia were not reported. Therefore, our findings should be interpreted cautiously. This meta-analysis of only 5 articles was too small to prove our hypothesis. The relationship between nuchal cord and the risk of ASD is not well delineated. Future studies with large sample sizes and longer follow-up periods will be required to draw better conclusions about the long-term effects of fetal nuchal cord and the risk of ASD.

\section{Conclusion}

Our results suggest that fetal nuchal cord is not a risk factor for ASD. Future large cohort studies should further investigate this relationship.

\section{Footnotes}

Conflicts of interest: No potential conflict of interest relevant to this article was reported.

Funding: We thank Hamadan University for its financial support with approval code 1400011035 .

\section{ORCID:}

Ensiyeh Jenabi $\odot$ https://orcid.org/0000-0002-4536-0814

Maryam Ahmadi 1 https://orcid.org/0000-0001-7616-1079

Azam Maleki @ https://orcid.org/0000-0001-7888-1985

\section{References}

1. Croen LA, Grether JK, Hoogstrate J, Selvin S. The changing prevalence of autism in California. J Autism Dev Disord 2002;32:207-15.

2. Williams E, Thomas K, Sidebotham H, Emond A. Prevalence and characteristics of autistic spectrum disorders in the ALSPAC cohort. Dev Med Child Neurol 2008;50:672-7.

3. Zhang X, Lv CC, Tian J, Miao RJ, Xi W, Hertz-Picciotto I, et al. Prenatal and perinatal risk factors for autism in China. J Autism Dev Disord 2010;40:1311-21.

4. Jenabi E, Bashirian S, Khazaei S. Association between neonatal jaundice and autism spectrum disorders among children: a meta-analysis. Clin Exp Pediatr 2020;63:8-13.

5. Jenabi E, Karami M, Khazaei S, Bashirian S. The association between preeclampsia and autism spectrum disorders among children: a metaanalysis. Korean J Pediatr 2019;62:126-30.

6. Jenabi E, Seyedi M, Hamzehei R, Bashirian S, Rezaei M, Razjouyan K, et al. Association between assisted reproductive technology and autism spectrum disorders in Iran: a case-control study. Clin Exp Pediatr 2020; 63:368-72.

7. Al-Ayadhi LY. Altered oxytocin and vasopressin levels in autistic children in Central Saudi Arabia. Neurosciences (Riyadh) 2005;10:47-50.

8. Najam S, Malik SE, Aqeel S, Rizwan N, Haider AR. Effect of nuchal cord on duration of labor. Int J Health Sci Res 2020;10:51-6.

9. Peesay M. Nuchal cord and its implications. Matern Health Neonatol Perinatol 2017;3:28.

10. Malla A, Pathak J, Shrestha S, Pant A, Shrivastava T, Amatya SM, et al. A study on parental age, pregnancy events and autism spectrum disorders. J 
Kathmandu Med Coll 2017;6:14-21.

11. Mamidala MP, Polinedi A, P T V PK, Rajesh N, Vallamkonda OR, Udani $\mathrm{V}$, et al. Prenatal, perinatal and neonatal risk factors of Autism Spectrum Disorder: a comprehensive epidemiological assessment from India. Res Dev Disabil 2013;34:3004-13.

12. Gardener H, Spiegelman D, Buka SL. Perinatal and neonatal risk factors for autism: a comprehensive meta-analysis. Pediatrics 2011;128:344-55.

13. Wang C, Geng H, Liu W, Zhang G. Prenatal, perinatal, and postnatal factors associated with autism: A meta-analysis. Medicine (Baltimore) 2017;96:e6696.

14. Ornoy A, Weinstein-Fudim L, Ergaz Z. Genetic syndromes, maternal diseases and antenatal factors associated with autism spectrum disorders (ASD). Front Neurosci 2016;10:316.

15. Page MJ, McKenzie JE, Bossuyt PM, Boutron I, Hoffmann TC, Mulrow CD, et al. Updating guidance for reporting systematic reviews: development of the PRISMA 2020 statement. J Clin Epidemiol 2021; 134:103-12.

16. Wells GA, Shea B, O'Connell D, Peterson J, Welch V, Losos M, et al. The Newcastle-Ottawa Scale (NOS) for assessing the quality of nonrandomised studies in meta-analyses [Internet]. Ottawa (ON): Ottawa Hospital Research Institute; 2017 [cited 2021 Feb 5]. Available from: http://www.ohri.ca/programs/clinical_epidemiology/oxford.asp.

17. Higgins JP, Thompson SG, Deeks JJ, Altman DG. Measuring inconsistency in meta-analyses. BMJ 2003;327:557-60.

18. Egger M, Davey Smith G, Schneider M, Minder C. Bias in meta-analysis detected by a simple, graphical test. BMJ 1997;315:629-34.

19. Glasson EJ, Bower C, Petterson B, de Klerk N, Chaney G, Hallmayer JF. Perinatal factors and the development of autism: a population study. Arch Gen Psychiatry 2004;61:618-27.
20. Piven J, Simon J, Chase GA, Wzorek M, Landa R, Gayle J, et al. The etiology of autism: pre-, peri- and neonatal factors. J Am Acad Child Adolesc Psychiatry 1993;32:1256-63.

21. ClappJF3rd,Lopez B, Simonean S. Nuchal cord and neurodevelopmental performance at 1 year. J Soc Gynecol Investig 1999;6:268-72.

22. Vasa R, Dimitrov R, Patel S. Nuchal cord at delivery and perinatal outcomes: single-center retrospective study, with emphasis on fetal acidbase balance. Pediatr Neonatol 2018;59:439-47.

23. Karabeg E, Karabeg E, Rešić B, Karabeg A. Can nuchal cord be responsible for later neurodevelopmental disorders? Int J Sci Eng Res 2018;9:1498503.

24. Huberman Samuel M, Meiri G, Dinstein I, Flusser H, Michaelovski A, Bashiri A, et al. general anesthesia may contribute to the association between cesarean delivery and autism spectrum disorder. J Autism Dev Disord 2019;49:3127-35.

25. Meldrum SJ, Strunk T, Currie A, Prescott SL, Simmer K, Whitehouse AJ. Autism spectrum disorder in children born preterm-role of exposure to perinatal inflammation. Front Neurosci 2013;7:123.

26. Jenabi E, Bashirian S, Khazaei S. Is breech presentation associated with autism spectrum disorders among children: a meta-analysis. Adv Human Biol 2019;9:12-5.

How to cite this article: Jenabi E, Ahmadi M, Maleki A. Is fetal nuchal cord associated with autism spectrum disorder? A meta-analysis. Clin Exp Pediatr 2022;65:131-5. https:/doi.org/ 10.3345/cep.2021.00514 